\title{
Multischeme Transport with Upwinding for the Rattlesnake Code
}

\author{
Yaqi Wang ${ }^{1}$,Mark Dehart ${ }^{2}$, Vincent Labouré ${ }^{2}$, Sebastian Schunert ${ }^{1}$, \\ Javier Ortensi ${ }^{2}$, Frederick Gleicher ${ }^{2}$ \\ ${ }^{1}$ Nuclear Engineering Methods and Development \\ Idaho National Laboratory \\ P.O. Box 1625 \\ Idaho Falls, ID 83415-3840 \\ ${ }^{2}$ Reactor Physics Design and Analysis \\ Idaho National Laboratory \\ P.O. Box 1625 \\ Idaho Falls, ID 83415-3840
}

January 2018

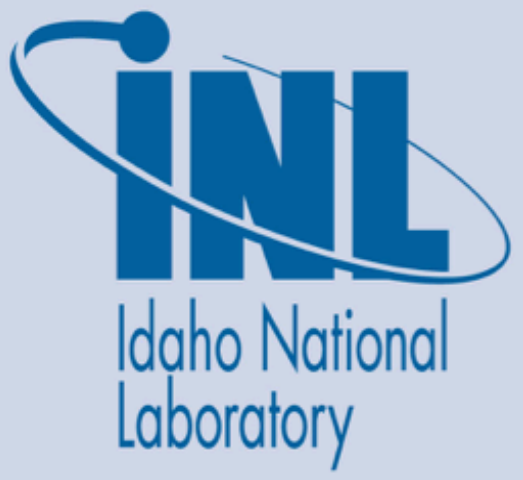

INL is a U.S. Department of Energy National Laboratory operated by Battelle Energy Alliance 


\section{DISCLAIMER}

This information was prepared as an account of work sponsored by an agency of the U.S. Government. Neither the U.S. Government nor any agency thereof, nor any of their employees, makes any warranty, expressed or implied, or assumes any legal liability or responsibility for the accuracy, completeness, or usefulness, of any information, apparatus, product, or process disclosed, or represents that its use would not infringe privately owned rights. References herein to any specific commercial product, process, or service by trade name, trade mark, manufacturer, or otherwise, does not necessarily constitute or imply its endorsement, recommendation, or favoring by the U.S. Government or any agency thereof. The views and opinions of authors expressed herein do not necessarily state or reflect those of the U.S. Government or any agency thereof. 


\section{Multischeme Transport with Upwinding for the Rattlesnake Code}

Yaqi Wang, Mark DeHart, Vincent Labouré, Sebastian Schunert, Javier Ortensi,
Frederick Gleicher

January 2018

Idaho National Laboratory

Nuclear Systems Design and Analysis

Idaho Falls, Idaho 83415

http://www.inl.gov

Prepared for the

U.S. Department of Energy

Office of Nuclear Energy

Under DOE Idaho Operations Office

Contract DE-AC07-05ID14517 


\begin{abstract}
This report describes efforts at INL to incorporate full support for a multischeme transport capability in Rattlesnake, the MOOSE-based multigroup radiation transport application. Specifically, this report details all five types of interface conditions for coupling six available discretization schemes within Rattlesnake using the upwinding method. These discretization schemes include $\mathrm{S}_{\mathrm{N}}$ (discrete ordinates method), $\mathrm{P}_{\mathrm{N}}$ (spherical harmonics expansion method) and diffusion for angular discretization, and continuous finite element method (CFEM) and discontinuous finite element method (DFEM) for spatial discretization. Another type of method based on Lagrange multipliers for coupling schemes with CFEM has been studied in [1]. In addtion to simple test problems, a more realistic application of multischeme transport for experiment simulations within the Transient Reactor Test Facility (TREAT) are presented. Several enhancements are reviewed at the end which could potentially be useful in the future.
\end{abstract}




\section{Contents}

$\begin{array}{lll}1 \text { Introduction } & 1\end{array}$

2 Theory 3

2.1 Transport equation and the upwinding interface conditions . . . . . . . 3

2.2 Multischeme upwinding interface conditions $\ldots \ldots \ldots \ldots \ldots$

2.2 .1 SN-SN interface condition $\ldots \ldots \ldots \ldots \ldots$

2.2 .2 PN-PN interface condition . . . . . . . . . . . . . . 7

2.2 .3 Diffusion-Diffusion interface condition . . . . . . . . . 7

2.2 .4 SN-PN interface condition . . . . . . . . . . . . . . . . . . 8

2.2 .5 SN-diffusion coupling . . . . . . . . . . . . . . . . . 9

2.2 .6 PN-Diffusion interface condition . . . . . . . . . . . 10

2.3 User interface and preconditioning . . . . . . . . . . . . . 10

3 Numerical Results 11

3.1 Pseudo one-dimensional test for SN-diffusion . . . . . . . . . . . . 11

3.2 Multischeme calculation for TREAT experiment . . . . . . . . . . . . . 14

3.2 .1 Motivation $\ldots \ldots \ldots \ldots \ldots \ldots \ldots \ldots$

3.2 .2 Model . . . . . . . . . . . . . . . . . . . 16

3.2 .3 Core Power Profile . . . . . . . . . . . . . . . . 18

3.2 .4 Discussion . . . . . . . . . . . . . . . . . . . . . 19

4 Conclusions and Future Work 20

References 22 


\section{Introduction}

Multischeme transport is a concept in which the spatial solution domain is divided into subdomains where various discretization schemes with different angular, space, energy resolutions can be applied [1-8]. It is noted that we stop using the name previously use in Rattlesnake methods development, multiscale, to avoid confusions in multiphysics environment, where multiscale is frequently used in materials modeling. The multischeme transport approach is motivated by the fact that a uniformly fine resolution over a full solution domain will require formidable computing resources for any realistic problems in the foreseeable future. On the other hand, such uniformly fine resolution is seldom required because of the possibility of obtaining certain coarse-level quantities of interest with homogenization techniques [9]. While homogenization techniques are sufficient for a variety of modeling and simulations tasks, they require representative geometries and homogenization equivalence, which may not be always available. There are also cases where fine quantities of interest are desired.

For example, the heat deposition in fuel pellets within a sophisticated experiment in the Transient Reactor Test Facility (TREAT) at Idaho National Laboratory (INL) is of significant interest, yet homogenization of the experiment cannot be done reliably and will not provide enough resolution for evaluating the heat deposition. Thus it makes sense to apply different discretization schemes or resolutions suitable for sub-domains. Computational resources can then be efficiently managed by concentrating the primarly efforts on the most important sub-domains while still taking into account the coupling with less important parts of the domain.

Multischeme transport is closely related with transport scheme adaptation [6, 8], in which schemes are applied on sub-domains adaptively based on a posterior indicator. Scheme adaptation assumes that homogenization equivalence has been done on the analytical transport equation and is thus scheme-independent. As a result, scheme boundaries does not have to be aligned with the boundary of any homogenization zones. In the current multischeme transport approach we do not consider scheme adaption, and let users decide where the scheme boundaries are. Multischeme transport also poses challenges on solving the resulting algebraic equation. It is desired that the multischeme equation is solved as a whole without iterations over the interface quantities.

This report focuses on multischeme implementation and verification within Rattlesnake [10], the MOOSE [11, 12]-based multigroup radiation transport application at INL. 
Rattlesnake is designed for both stand-alone and multiphysics simulations. It possesses all of the features available within the MOOSE framework, including unstructured higherorder meshes, massive parallelization, dimension agnosticism, etc. Rattlesnake solves steady-state, transient and eigenvalue problems. Its applications to fully-coupled multiphysics simulation and the tightly-coupled multiphysics simulations with data transfer have been both successfully demonstrated [13-16]. Rattlesnake provides various discretization schemes, including the self-adjoint angular flux (SAAF) and least squares (LS) formulations with continuous finite element method (CFEM), the first-order formulation with discontinuous finite element method (DFEM), all detailed in [10]. Angular discretization can be applied with the discrete ordinates method (SN), the spherical harmonics expansion method (PN) or using the diffusion approximation. To enable a multischeme capability and leverage all these schemes, we need to execute interface coupling in space, angle and energy properly. In this report, we assume embedded energy structures across two neighboring schemes, i.e. any group in these two scales is either a subset or completely outside of another group. Flux prolongation and projection with a different number of energy groups has been implemented in Rattlesnake for the purpose of on-the-fly group condensation with the method in Ref. [17], but has not been applied on multischeme interface conditions. Spatial coupling is implemented via general mortar FEM and the interface kernel capability in MOOSE, both of which have found applications in other disciplines, and thus will not be included in this report. This report presents the interface conditions for coupling various angular discretization methods: SN, PN and diffusion. Weak forms of these interface conditions will be discussed. The interface condition for coupling SN-PN, or hybrid SN-PN has been studied in the past [1-8]. This report includes the SN-PN interface condition with upwinding that was developed in [1] for completeness and extend to the cases of all other coupling types except with LS schemes. Interface conditions for LS schemes will need to be imposed slightly differently [10] and thus will not be considered in this report. Although users are allowed to amend the interface conditions with the properties defined on interfaces, such as discontinuity factors from the generalized homogenization equivalence theory [9], this report will only discuss the nominal conditions. It is noted that steady-state, transient and eigenvalue problems can be solved with or without the multischeme transport by Rattlesnake.

In Section 2, we first introduce the notations and basic background with the steady-state one-group transport equation with isotropic scattering. Although the equations are given for this simplified transport equation, nothing prevents us from extending the interface conditions to more complicated cases with multigroup, transient, anisotropic scattering, etc. We also present the analytical interface condition with upwinding scheme in the weak form without angular discretization. We then apply various combinations of angular discretiza- 
tions on two sides to derive the particular interface conditions. In Section 3, we present numerical results for $\mathrm{SN}$-diffusion with the example presented previously in [1]. We then apply the multischeme transport for modeling a TREAT experiment. The experiment region is modeled with SN and fine spatial resolution while the rest of the core is modeled with diffusion with assembly homogenized SPH (superhomogenization)-corrected cross sections [atreatreport]. Conclusions are drawn and potential future work is discussed in Section 4.

\section{Theory}

\subsection{Transport equation and the upwinding interface conditions}

The one-group steady-state transport equation is:

$$
\vec{\Omega} \cdot \vec{\nabla} \Psi+\Sigma_{t}(\vec{x}) \Psi(\vec{x}, \vec{\Omega})=\Sigma_{S}(\vec{x}) \Phi(\vec{x})+S(\vec{x}, \vec{\Omega}),
$$

where the spatial position $\vec{x} \in \mathscr{D}$ and direction of motion $\vec{\Omega} \in \mathscr{S}$ are independent variables and $\mathscr{D}$ and $\mathscr{S}$ are the spatial domain and the unit sphere respectively, and where $\Psi$, the angular flux, is to be sought. $\Phi$ is defined as $\frac{\int_{\mathscr{S}} \Psi\left(\vec{x}, \vec{\Omega}^{\prime}\right) \mathrm{d}[\Omega]^{\prime}}{\int_{\mathscr{S}} \mathrm{d}[\Omega]^{\prime}} . S$ is the external source. $\Sigma_{t}$ and $\Sigma_{s}$ are the total and scattering cross sections. The boundary $\partial \mathscr{D}$ is vacuum with

$$
\Psi(\vec{x}, \vec{\Omega})=0, \vec{x} \in \partial \mathscr{D}, \vec{\Omega} \cdot \vec{n}_{\mathrm{b}}(\vec{x})<0,
$$

where $\vec{n}_{\mathrm{b}}$ is the unit outward normal on the boundary. Multischeme transport relies on splitting the solution domain $\mathscr{D}$ into several non-overlapping subdomains $\mathscr{D}_{i}, i=1, \cdots, N$, where $N$ is the number of subdomains, as illustrated in Fig. 11. The union of all subdomains covers the entire solution domain, i.e. $\mathscr{D}=\cup_{i=1}^{N} \mathscr{D}_{i}$. The subdomain interface is defined as $\Gamma=\cup_{1 \leq i \leq N-1, i<j \leq N} \mathscr{D}_{i} \cap \mathscr{D}_{j}$. Discretization schemes are applied on each individual subdomain and then joined together with interface conditions. It is noted that all interface conditions in this report can be applied to transient or eigenvalue problems with anisotropic scattering and various types of boundary conditions. In mulitgroup calculations, interface conditions are applied to the fluxes of each group independently. The Lagrange multiplier interface conditions with continuous FEM (CFEM) have been presented in Ref. [1]. We therefore will only focus on the upwinding interface condition. This report can be considered as an extension of Ref. [1] in the sense that all interface conditions with upwinding 


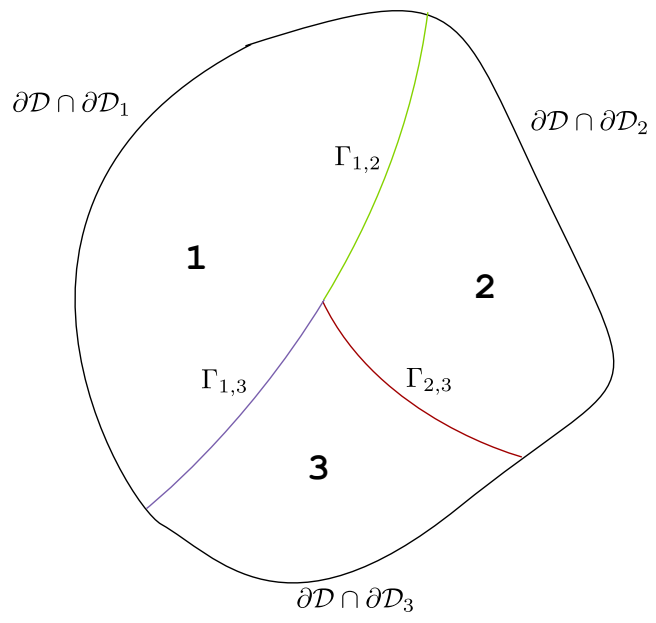

Figure 1: Domain decomposition with three subdomains.

for coupling all schemes are detailed including the interface conditions for hybrid SN-PN calculations.

The upwinding method solves the transport equation for any streaming direction and for each subdomain with surface source boundary conditions provided by its neighbors at the upwinding location (i.e. upstream) of the boundary with respect to the streaming direction:

$$
\begin{aligned}
& \Psi^{+}(\vec{x}, \vec{\Omega})=\Psi^{-}(\vec{x}, \vec{\Omega}), \vec{x} \in \Gamma, \vec{\Omega} \cdot \vec{n}>0, \\
& \Psi^{-}(\vec{x}, \vec{\Omega})=\Psi^{+}(\vec{x}, \vec{\Omega}), \vec{x} \in \Gamma, \vec{\Omega} \cdot \vec{n}<0,
\end{aligned}
$$

where unit norm $\vec{n}$ is defined on $\Gamma$. It is perpendicular to $\Gamma$ and depends on the position $\vec{x}$. Its orientation does not affect the interface condition. The \pm superscript on the angular flux is used to denote the quantity on the neighboring side of $\Gamma$ with respect to $\vec{n}$ :

$$
\left.a(\vec{x})^{ \pm}\right|_{\vec{x} \in \Gamma} \equiv \lim _{s \rightarrow 0^{+}} a(\vec{x} \pm s \vec{n})
$$

where $a$ is a generic function defined over the solution domain $\mathscr{D}$.

The analytical interface condition with upwinding can be written in its weak formulation as

$$
-\left\langle\left[\left[\Psi^{*}\right]\right], \Psi^{-}\right\rangle_{\Gamma}^{+}+\left\langle\left[\left[\Psi^{*}\right]\right], \Psi^{+}\right\rangle_{\Gamma}^{-}
$$


with the following definitions:

$$
\begin{gathered}
\llbracket[a(\vec{x})]||_{\vec{x} \in \Gamma} \equiv a^{+}-a^{-} \\
\langle a(\vec{x}, \vec{\Omega}), b(\vec{x}, \vec{\Omega})\rangle_{\Gamma}^{+} \equiv \int_{\Gamma} \int_{\vec{\Omega} \cdot \vec{n}>0}|\vec{\Omega} \cdot \vec{n}| a(\vec{x}, \vec{\Omega}) b(\vec{x}, \vec{\Omega}) \mathrm{d}[\Omega] \mathrm{d} \\
\langle a(\vec{x}, \vec{\Omega}), b(\vec{x}, \vec{\Omega})\rangle_{\Gamma}^{-} \equiv \int_{\Gamma} \int_{\vec{\Omega} \cdot \vec{n}<0}|\vec{\Omega} \cdot \vec{n}| a(\vec{x}, \vec{\Omega}) b(\vec{x}, \vec{\Omega}) \mathrm{d}[\Omega] \mathrm{d} .
\end{gathered}
$$

LS schemes need to have the following weak formulation

$$
\left\langle\Psi^{*+},[[\Psi]]\right\rangle_{\Gamma}^{+}-\left\langle\Psi^{*-},[[\Psi]]\right\rangle_{\Gamma}^{-} .
$$

One can mix and match Eq. (5a) for coupling LS schemes with other schemes but we will not consider upwinding couplings with LS schemes in this report.

\subsection{Multischeme upwinding interface conditions}

Currently, Rattlesnake supports eight discretization schemes: SAAF-CFEM-SN [18], SAAFCFEM-PN [19], LS-CFEM-SN [20], LS-CFEM-PN [21], DFEM (discontinuous finite element method for the first-order formulation)-SN [22], DFEM-PN [23], CFEM-Diffusion and DFEM-Diffusion. We apply different combinations of angular discretization schemes: $\mathrm{SN}, \mathrm{PN}$ and diffusion, to obtain the multischeme interface conditions (with the exception of the two LS schemes). SN schemes require an angular quadrature $\left\{\vec{\Omega}_{m}, w_{m}, m=1, \cdots, M\right\}$, where $M$ is the number of directions in the quadrature set. This angular quadrature needs to satisfy certain properties such as exact integration of the spherical harmonics up to the scattering degree for conservation. The transport equation is solved on discrete ordinate space (collocation method) and all angular integrations are replaced with a quadrature formulation. PN schemes expand the angular flux with spherical harmonics up to a certain degree $L$.

$$
\Psi(\vec{x}, \vec{\Omega})=\sum_{l=0}^{L} \sum_{m=-l}^{l} R_{l, m}(\vec{\Omega}) \Phi_{l, m}(\vec{x})
$$

where

$$
\Phi_{l, m}(\vec{x}) \equiv \int_{\mathscr{S}} \Psi(\vec{x}, \vec{\Omega}) Y_{l, m}(\vec{\Omega}) d \Omega
$$

$Y_{l, m}$ are the real spherical harmonics and $R_{l, m}$ are their conjugates, satisfying 


$$
\int_{\mathscr{S}} R_{n, k} \vec{\Omega} Y_{l, m}(\vec{\Omega}) d \Omega=\delta_{n, l} \delta_{k, m}
$$

Eq. (7) can be written in a shorter vector notation as $\Psi=\mathbf{R}^{T} \mathbf{m}$. It is noted that some angular moments are zero in reduced problem dimensions due to symmetry and can be removed from the expansion. The diffusion approximation assumes the Fick's law: $\mathbf{J}=\mathrm{D} \vec{\nabla} \Phi$, where the current $\mathbf{J}=\left\{\Phi_{1,0}, \Phi_{1,1}, \Phi_{1,-1}\right\}$, thus the scalar flux $\Phi$ can be solved independently from other angular moments. For notation simplicity, we define a new operator

$$
(a(\vec{x}), b(\vec{x}))_{\Gamma} \equiv \int_{\Gamma} a(\vec{x}) b(\vec{x}) \mathrm{d} .
$$

It is noted that we use $\Gamma$ to denote the interface between two schemes although more than two schemes are allowed in multischeme transport calculations and each interface between two schemes will be a subset of Gamma.

\subsubsection{SN-SN interface condition}

The SN-SN interface condition is:

$$
-\sum_{\vec{\Omega}_{m} \cdot \vec{n}>0} w_{m}\left(\left|\vec{\Omega}_{m} \cdot \vec{n}\right|\left[\left[\Psi_{m}^{*}\right]\right], \Psi_{m}^{-}\right)_{\Gamma}+\sum_{\vec{\Omega}_{m} \cdot \vec{n}<0} w_{m}\left(\left|\vec{\Omega}_{m} \cdot \vec{n}\right|\left[\left[\Psi_{m}^{*}\right]\right], \Psi_{m}^{+}\right)_{\Gamma}
$$

This interface condition enables coupling with SAAF-CFEM-SN, LS-CFEM-SN and DFEM$\mathrm{SN}$. When coupling SN with SN, we currently require the two sides to have the same angular quadrature. We have not yet enabled the general angular interpolation in the angular quadratures although some of the basic capability has been implemented for evaluating angular derivatives [16]. If both sides are DFEM-SN, the interface condition makes the multischeme on the two connecting subdomains equivalent to applying the same single DFEM-SN on the two subdomains. The upwinding condition restores the causality inherent to the hyperbolic nature of particle transport on the subdomain interface for CFEM, and thus shows significant improvement on the solution accuracy for problems with interfaces having a sharp transition of total cross sections [24]. 


\subsubsection{PN-PN interface condition}

If we insert $\Psi=\mathbf{R}^{T} \mathbf{m}$ into Eq. 5 a we can see that

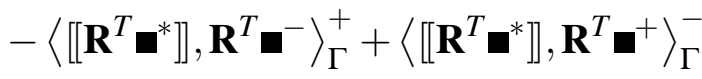

$$
\begin{aligned}
& =-\left(\left[\left[\mathbf{\varpi}^{*}\right]\right], \mathbf{L}^{+} \mathbf{\square}^{-}\right)_{\Gamma}+\left(\left[\left[\mathbf{\varpi}^{*}\right]\right], \mathbf{L}^{-} \mathbf{\square}^{+}\right)_{\Gamma} \\
& =\left(\left[\left[\boldsymbol{\varpi}^{*}\right]\right], \frac{\mathbf{L}^{+}+\mathbf{L}^{-}}{2}[[\mathbf{\square}]]-\mathbf{L}\{\{\boldsymbol{\square}\}\}\right)_{\Gamma}
\end{aligned}
$$

where

$$
\begin{aligned}
\mathbf{L}^{+} & \equiv \int_{\vec{\Omega} \cdot \vec{n}>0} \overrightarrow{\mathbf{R}}(\vec{\Omega}) \overrightarrow{\mathbf{R}}^{T}(\vec{\Omega})|\vec{\Omega} \cdot \vec{n}| d \Omega, \\
\mathbf{L}^{-} & \equiv \int_{\vec{\Omega} \cdot \vec{n}<0} \overrightarrow{\mathbf{R}}(\vec{\Omega}) \overrightarrow{\mathbf{R}}^{T}(\vec{\Omega})|\vec{\Omega} \cdot \vec{n}| d \Omega \\
\mathbf{L} & \equiv \int_{\mathscr{S}} \overrightarrow{\mathbf{R}}(\vec{\Omega}) \overrightarrow{\mathbf{R}}^{T}(\vec{\Omega}) \vec{\Omega} \cdot \vec{n} d \Omega=\mathbf{L}^{+}-\mathbf{L}^{-}
\end{aligned}
$$

and average operator

$$
\{\{a(\vec{x})\}\}_{\vec{x} \in \Gamma} \equiv \frac{a^{+}+a^{-}}{2} .
$$

$\mathbf{L}, \mathbf{L}^{+}$and $\mathbf{L}^{-}$are symmetric matrices. We emphasize that although these $\mathbf{L}$-matrices depend on $\vec{n}$, their eigen-structures do not, and are thus rotational invariant. L-matrices will not be square if two sides have different spherical harmonics truncation order. Another option resulting in a sparser moment coupling matrix is to use a Lax-Friedrich numerical flux which yields the following interface terms [25, 26]:

$$
\left(\llbracket \mathbf{\varpi}^{*} \rrbracket, \frac{1}{2}\|\vec{n}\|_{1} \mathbf{P} \llbracket \mathbb{\square} \rrbracket-\mathbf{L}\{\{\mathbf{\square}\}\}\right)_{\Gamma_{\mathrm{int}}},
$$

with $\mathbf{P} \equiv \int_{\mathscr{S}} \mathbf{R} \mathbf{R}^{T} \mathrm{~d}[\Omega]$ and $\|\cdot\|_{1}$ is the $\mathrm{L}_{1}$ norm of a vector.

\subsubsection{Diffusion-Diffusion interface condition}

We apply the interior penalty method [27]:

$$
\left(\left[\left[\Phi^{*}\right]\right], \kappa[[\Phi]]\right)_{\Gamma}+\left(\left[\left[\Phi^{*}\right]\right],\{\{\mathrm{D} \vec{\nabla} \Phi \cdot \vec{n}\}\}\right)_{\Gamma}+\varepsilon\left(\left\{\left\{\mathrm{D} \vec{\nabla} \Phi^{*} \cdot \vec{n}\right\}\right\},[[\Phi]]\right)_{\Gamma} .
$$


$\varepsilon$ can be $-1,0$, or 1 , corresponding to non-symmetric, incomplete and symmetric interior penalty methods. We use the following equation for the penalty coefficient $\kappa$ :

$$
\kappa=\left\{\left\{2 p(p+1) \frac{\mathrm{D}}{h_{\perp}}\right\}\right\},
$$

where $p$ is the polynomial order of the element shape functions; $h_{\perp}$ is the length of the element orthogonal to the side. It is noted that the PN-PN interface condition, Eq. (11), with $\mathrm{P}_{1}$ on both side with Fick's law is equivalent to the incomplete interior penalty method with constant $\kappa=\frac{1}{4}$.

\subsubsection{SN-PN interface condition}

This condition has been presented in [1]. We provide it here for completeness. To simplify the notation, we adapt our convention, i.e. make the unit normal vector on the interface $\vec{n}$ always point towards the $S_{N}$ side. Thus we can drop the plus and minus superscript without creating confusion. The $S_{N}$ side form is

$$
\sum_{\vec{\Omega}_{m} \cdot \vec{n}<0} w_{m}\left(\left|\vec{\Omega}_{m} \cdot \vec{n}\right| \Psi_{m}^{*}, \Psi_{m}\right)_{\Gamma}-\sum_{\vec{\Omega}_{m} \cdot \vec{n}>0} w_{m}\left(\left|\vec{\Omega}_{m} \cdot \vec{n}\right| \Psi_{m}^{*}, \mathbf{R}^{T}\left(\vec{\Omega}_{m}\right) \boldsymbol{\square}\right)_{\Gamma} .
$$

The $P_{N}$ side form is

$$
\left(\boldsymbol{\bullet}^{*}, \overline{\mathbf{L}}^{+}(\vec{n}) \mathbf{\square}\right)_{\Gamma}-\left(\boldsymbol{\varpi}^{*}, \mathbf{j}\right)_{\Gamma}
$$

where

$$
\begin{aligned}
\overline{\mathbf{L}}^{+}(\vec{n}) & \equiv \sum_{\vec{\Omega}_{m} \cdot \vec{n}>0} w_{m} \mathbf{R}\left(\vec{\Omega}_{m}\right) \mathbf{R}^{T}\left(\vec{\Omega}_{m}\right)\left|\vec{\Omega}_{m} \cdot \vec{n}\right| \\
\mathbf{j} & \equiv \sum_{\vec{\Omega}_{m} \cdot \vec{n}<0} w_{m}\left|\vec{\Omega}_{m} \cdot \vec{n}\right| \mathbf{R}\left(\vec{\Omega}_{m}\right) \Psi_{m}
\end{aligned}
$$

Numerical results indicate that the discrete matrix $\overline{\mathbf{L}}^{+}$gives better solutions than the analytical matrix $\mathbf{L}^{+}$. It is noted that eigenvalues of the discrete version $\overline{\mathbf{L}}^{+}$of $\mathbf{L}^{+}$depends on $\vec{n}$.

It is interesting to check the equivalence of SN and PN for the interface terms. Suppose we have the direction-to-moment matrix $\mathbf{D}$, constructed from angular quadrature (to convert 
angular fluxes to flux moments) and let the moment-to-direction matrix be $\mathbf{M}=\mathbf{D}^{-1}$. We then denote the $m$-th row of $\mathbf{M}$ as $\vec{M}_{m}^{T}$, and we have the following

$$
\begin{aligned}
& \sum_{\vec{\Omega}_{m} \cdot \vec{n}<0} w_{m}\left(\left|\vec{\Omega}_{m} \cdot \vec{n}\right| \Psi_{m}^{*}, \Psi_{m}\right)_{\Gamma} \\
= & \left(\mathbf{D} \boldsymbol{\varpi}^{*},\left(\sum_{\vec{\Omega}_{m} \cdot \vec{n}<0} w_{m}\left|\vec{\Omega}_{m} \cdot \vec{n}\right| \vec{M}\left(\vec{\Omega}_{m}\right) \vec{M}^{T}\left(\vec{\Omega}_{m}\right)\right) \mathbf{D} \boldsymbol{\square}\right)_{\Gamma} \\
= & \left(\mathbf{\square}^{*}, \hat{\mathbf{L}}^{-} \mathbf{\square}\right)_{\Gamma} .
\end{aligned}
$$

If we consider all the other terms and replace $\vec{M}_{m}$ with $\vec{R}\left(\vec{\Omega}_{m}\right)$, we retrieve the above SNPN condition. This provides another justification on the choice of discrete $\mathbf{L}$-matrix instead of the analytical one. It also suggests that we could replace $\mathbf{R}\left(\vec{\Omega}_{m}\right)$ in Eq. $(19)$, Eq. (21), Eq. 22) with $\vec{M}_{m}$.

\subsubsection{SN-diffusion coupling}

We apply the Robin boundary condition on the diffusion side,

$$
\frac{1}{4} \Phi-\frac{1}{2 e} \mathrm{D} \vec{\nabla} \Phi \cdot \vec{n}=J^{+}
$$

where

$$
J^{+}=\sum_{\vec{\Omega}_{m} \cdot \vec{n}<0} w_{m}\left|\vec{\Omega}_{m} \cdot \vec{n}\right| \Psi_{m},
$$

and $e$ is an extrapolation factor for better diffusion solution further away from the interface. This gives the terms

$$
\frac{e}{2}\left(\Phi^{*}, \Phi\right)_{\Gamma}-2 e\left(\Phi^{*}, J^{+}\right)_{\Gamma}
$$

We apply the surface source boundary condition on the SN side,

$$
\Psi_{m}=\frac{\Phi}{4 \pi}-\frac{3}{4 \pi} \mathrm{D} \vec{\nabla} \Phi \cdot \vec{\Omega}_{m}, \vec{\Omega}_{m} \cdot \vec{n}>0
$$

which results into the following terms

$$
\left.\sum_{\vec{\Omega}_{m} \cdot \vec{n}>0} w_{m}\left(\left|\vec{\Omega}_{m} \cdot \vec{n}\right| \Psi_{m}^{*}, \Psi_{m}\right)_{\Gamma}-\sum_{\vec{\Omega}_{m} \cdot \vec{n}>0} w_{m}\left(\left|\vec{\Omega}_{m} \cdot \vec{n}\right| \Psi_{m}^{*}, \frac{\Phi}{4 \pi}-\frac{3}{4 \pi} \mathrm{D} \vec{\nabla} \Phi \cdot \vec{\Omega}_{m}\right)\right)_{\Gamma}
$$


For $\mathrm{SN}-\mathrm{P}_{0}$, on $\mathrm{P}_{0}$ side, $\mathbf{L}^{+}=\frac{1}{4 \pi} \frac{1}{4}$ and $\vec{j}=\frac{1}{4 \pi} J^{+}$. Thus $\mathrm{SN}-\mathrm{P}_{0}$ condition is equivalent with $e=\frac{1}{2}$ with $\frac{1}{4 \pi}$ as the scaling factor for the scalar flux. The original Robin condition has $e=1$. The vacuum boundary condition with asymptotic analysis gives $e=\frac{2}{3 \times 0.710446}=$ 0.938838 [28]. On SN side, the terms are almost identical with Eq. (30) except the extra current term boxed in Eq. (30).

\subsubsection{PN-Diffusion interface condition}

Currently, PN-Diffusion is treated the same as $\mathrm{PN}-\mathrm{P}_{0}$.

\subsection{User interface and preconditioning}

In summary, we have presented five types of interface conditions for coupling six schemes in Rattlesnake. Subdomains are specified through blocks of a mesh, which can be either generated separately or by using one of the built-in mesh generation capabilities. Users need to describe the transport equation with the particle type (currently Rattlesnake supports neutron and thermal radiation), equation type (source, eigenvalue or transient), number of groups, boundary conditions, volumetric or boundary sources if any. Cross sections are given in separate material input blocks. Then users assign transport schemes to mesh blocks for multischeme transport. All the necessary kernels, boundary conditions, etc. for setting up the equation system will be added by Rattlesnake behind the scene depending on the scheme and the availability of cross sections. Interface conditions are also automatically added. A typical input for doing multischeme transport calculation can be found in Section 3. Rattlesnake uses the PJFNK (preconditioned Jacobian-free Newton Krylov) solver to solve the algebraic equations for steady-state source or eigenvalue problems and a time step of transient problems. The default preconditioning for multischeme transport is to precondition all transport schemes independently. Users can control how the preconditioning matrices are to be assembled, for example, whether the scattering term is included or not. Users can also choose various preconditioning techniques made available through PETSc [29] and few scheme-specific preconditioners, such as sweeper preconditioner, etc. 


\section{Numerical Results}

All interface conditions were tested and regression tests were added in Rattlesnake. We will present one simple pseudo one-dimensional test first to verify the correctness of the SN-diffusion condition because it is the one we are going to use for TREAT experiment modeling. Preliminary TREAT experiment results with SN-diffusion are given in the second example.

\subsection{Pseudo one-dimensional test for $\mathrm{SN}$-diffusion}

The setting of this problem is the same as the one in [1]. It is a simple one-group homogeneous fixed-source problem. The domain is $8 \mathrm{~cm} \times 8 \mathrm{~cm}$. Total and scattering cross sections are uniform, $1 \mathrm{~cm}^{-1}$ and $0.9 \mathrm{~cm}^{-1}$ respectively, and the external source is isotropic and uniform with $1 \mathrm{~cm}^{-3} \mathrm{~s}^{-1}$. Left, right and top boundaries are reflecting and the bottom boundary is vacuum. These settings make the problem pseudo one-dimensional, i.e. the scalar flux without discretization error is constant along the lines with $y=$ const. The problem is illustrated in Fig. 2. We also divided the domain into two subdomains by the line

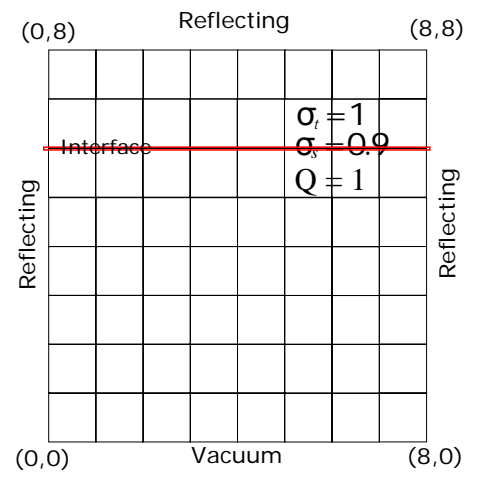

Figure 2: A one-group homogeneous problem.

starting through $(0,6) \mathrm{cm}$ and $(6,6) \mathrm{cm}$ and evaluated the integral of the scalar flux on these two subdomains as $R_{u}=\int_{\mathscr{D}_{P N}} \Phi \mathrm{d}$ and $R_{l}=\int_{\mathscr{D}_{S N}} \Phi \mathrm{d}$ respectively. We applied SN on the bottom subdomain with level-symmetric angular quadrature of order 16 and diffusion on the top subdomain. Linear Lagrange shape functions were used. The errors of the integrals of the scalar flux on both subdomains with different extrapolation factor $e$ were plotted in Fig. 3. We used the solution with S16 on the entire solution domain as the reference. 


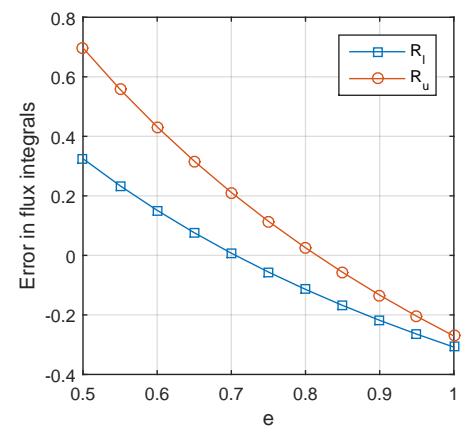

Figure 3: Error of flux integrals with $e$ factor.

The reference values for $R_{u}$ and $R_{l}$ are $378.978118 \mathrm{~s}^{-1}$ and $156.316625 \mathrm{~s}^{-1}$ respectively. It can be seen that the $e$ factor affects the solutions on both side. And we can adjust the factor to make certain quantities of interest to better match the reference values. How this factor is generated for a particular problem is possibly problem dependent and an open question.

The full input for this problem is

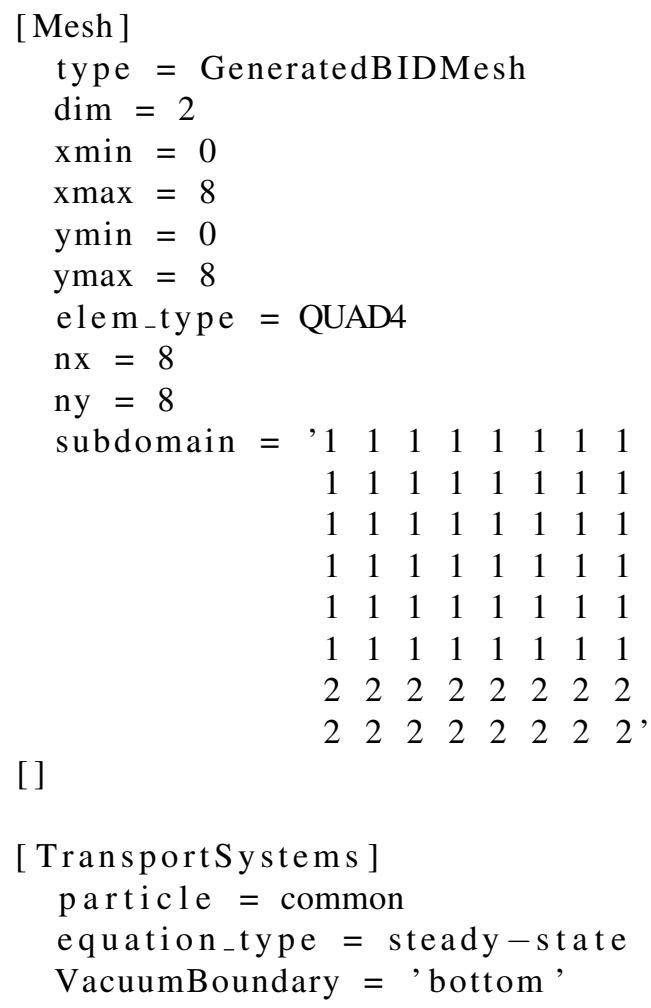




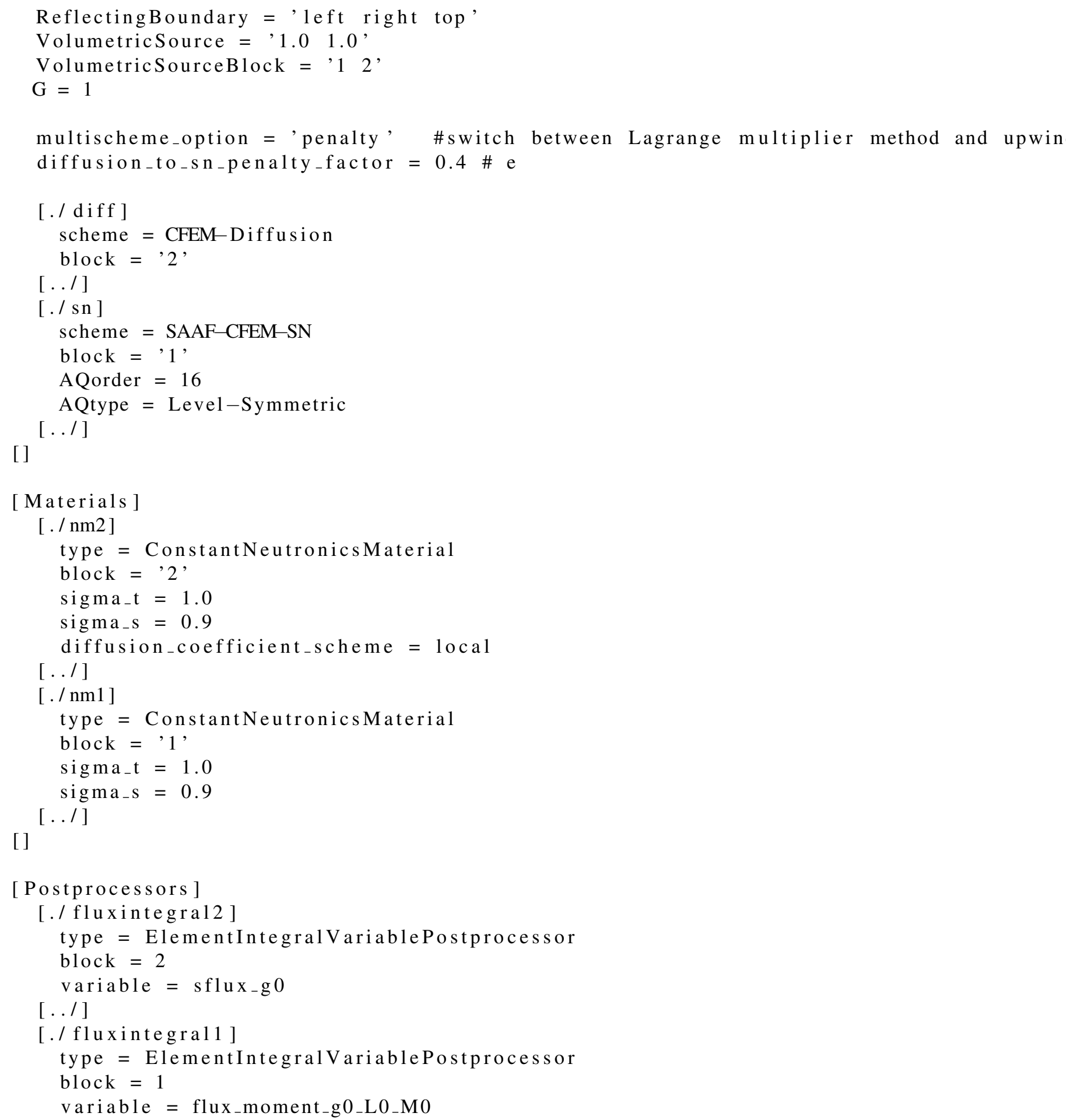




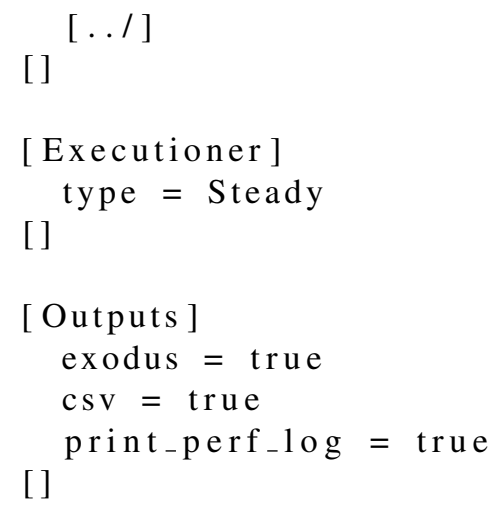

The calculation can be done in a second on a typical desktop with one processor.

\subsection{Multischeme calculation for TREAT experiment}

\subsubsection{Motivation}

In this section, we present the results for a more practical problem: the Multi-SERTTA (Static Environment Rodlet Transient Test Apparatus) experiment. This name refers to a experiment vehicle originally planned to be tested in TREAT in 2018. It consists of four units stacked on top of each other, each unit containing a rodlet with a stack of fuel pellets. Each stack is surrounded by a borated steel filter, so as to try to equalize the energy deposition in each stack. 


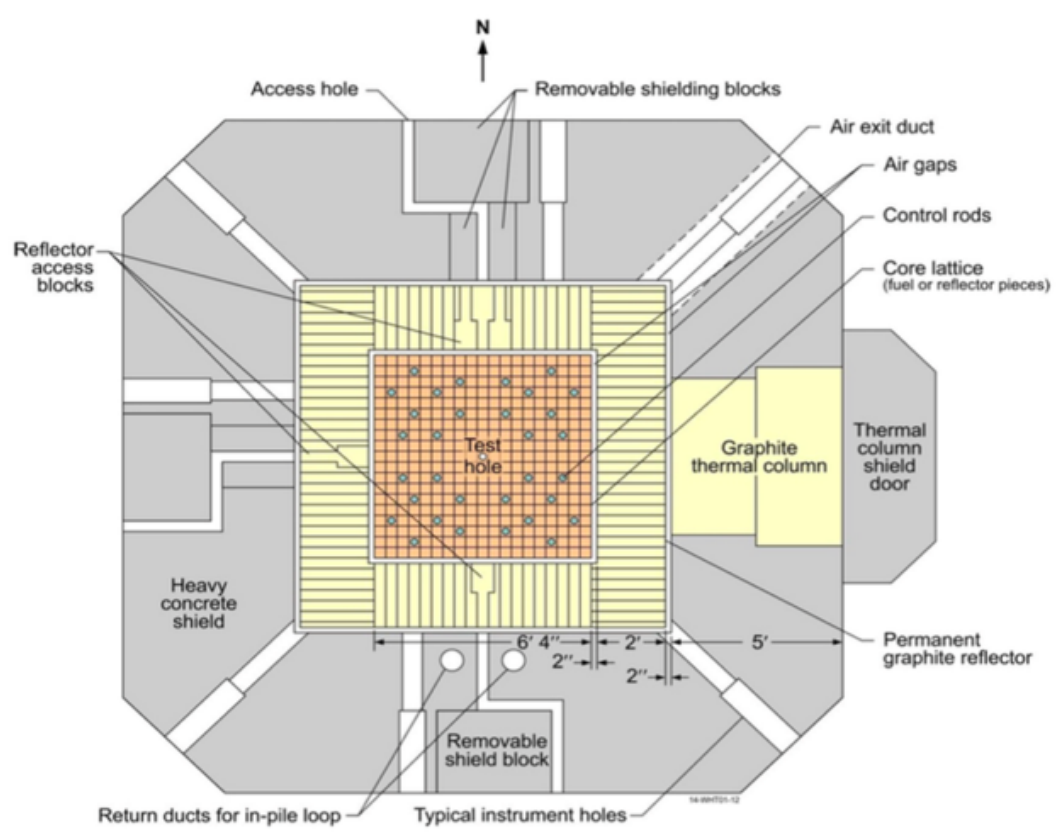

Figure 4: Top schematic view of the TREAT reactor. 

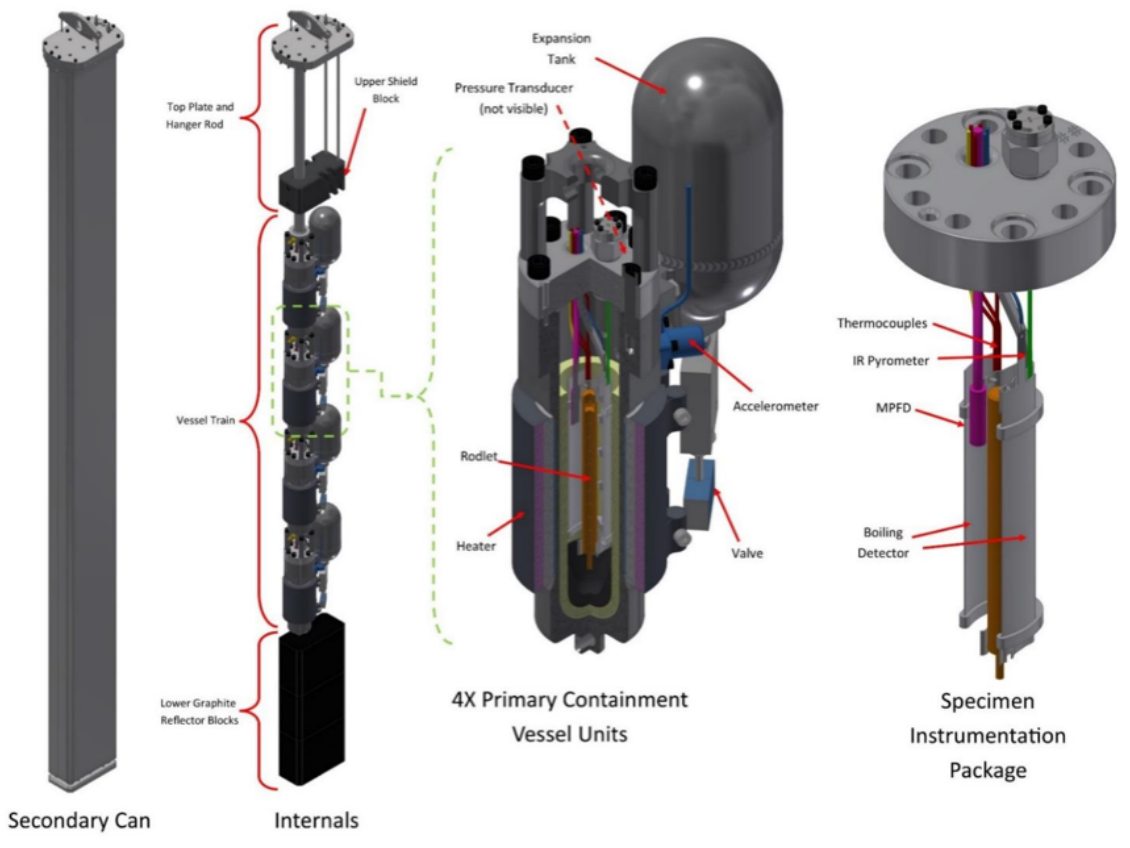

Figure 5: Description of the Multi-SERTTA experiment.

The experiment is conducted as follows: starting from a steady state with a low power, a control rod is rapidly withdrawn, thereby inserting a large amount of reactivity. The power in the core then dramatically increases up to several Gigawatts in less than a second. The negative temperature feedback of the core ensures that the power decreases to values very small compared to the peak power.

\subsubsection{Model}

This problem has many challenges, including many asymmetries, void regions and large complexity in the geometry, making it particularly hard to fully converge with a traditional transport solver. Fig. 4 gives a top view of the core. Since the experimenters need to be able to determine the energy deposited in each stack, the main interest in this problem is the experiment region. As a result, it is natural to want to use a multischeme approach with a high-fidelity method for that particular region and less accuracy for the rest of the core. Fig. 6 show the models for both the full-core and the experiment region. In particular, it clearly appears that the experiment region is considerably smaller than the rest of the core 
and thus that a multischeme calculation would better optimize the computational resources than running a full-core transport calculation.

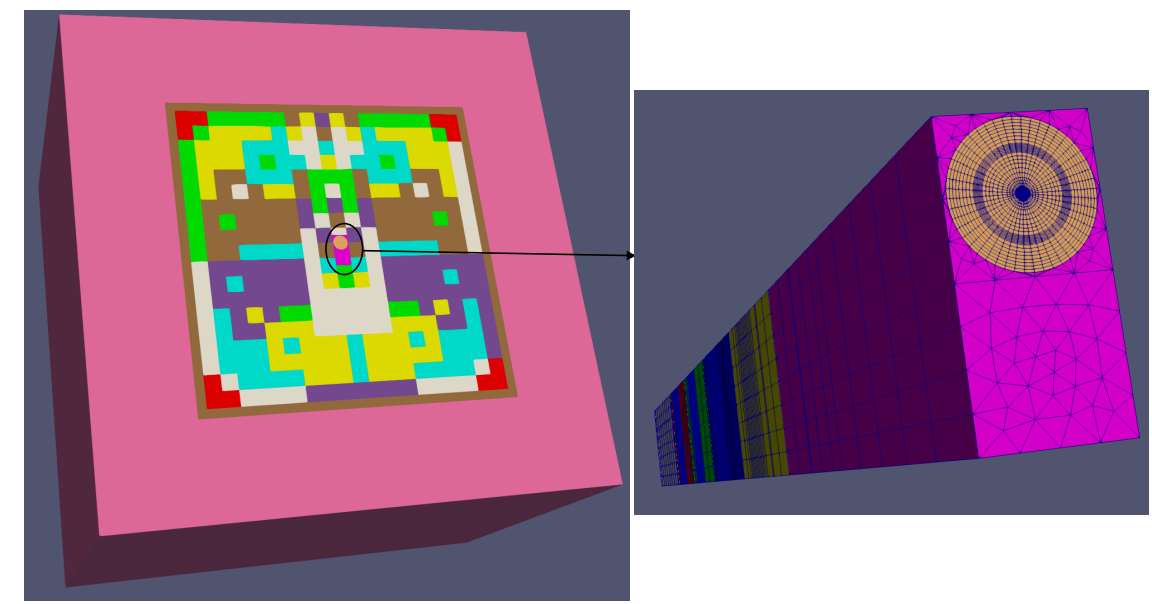

Figure 6: Left: numerical model for the TREAT core. Right: zoom on the experiment region (much more spatially limited).

This calculation leverages the multiphysics capabilities of MAMMOTH, which couples neutronics and temperature feedback. For now, only an adiabatic model is used but will be replaced with a direct coupling with Bison in the near future.

We then use a SAAF-CFEM-SN scheme for the experiment region and a CFEM-Diffusion scheme elsewhere. Table 1 compares the number of unknowns for that particular model, both for a high-fidelity transport scheme everywhere, diffusion scheme everywhere and the multischeme approach as a function of the quadrature order $N$ and clearly shows the benefits of the latter: the number of unknowns compared to full-core transport is greatly reduced.

In addition, it is noted that for the parts of the core using Diffusion (either full-core or multischeme), SPH correction has been applied to the cross-section to match the reference reaction rates from Serpent [30]. This means that the multischeme solution without equivalence (SPH or Discontinuity Factors) may not necessarily be superior to SPH-corrected diffusion, if the homogenization error is large. This could however be addressed by using equivalence techniques with the multischeme approach. 
Table 1: Number of unknowns for the TREAT MSERTTA model for different numerical schemes (for different quadrature orders $N$ ). The diffusion row has only one column since it does not depend on the quadrature order.

\begin{tabular}{|c|ccc|}
\hline & \multicolumn{3}{|c|}{ Quadrature Order $N$} \\
\hline & 2 & 4 & 6 \\
\hline Diffusion & \multicolumn{3}{|c|}{$9.5 \times 10^{6}$} \\
\hline SAAF-SN (full-core) & $7.3 \times 10^{7}$ & $2.2 \times 10^{8}$ & $4.4 \times 10^{8}$ \\
\hline Multischeme SAAF-SN/Diffusion & $2.2 \times 10^{7}$ & $4.9 \times 10^{7}$ & $9.0 \times 10^{7}$ \\
\hline
\end{tabular}

\subsubsection{Core Power Profile}

Fig. 7 shows the power profile in the core for the SPH full-core Diffusion scheme and the multischeme Diffusion/SAAF-CFEM-SN (for S2 and S4) with SPH corrected crosssections only used outside the experiment region. While the two multischeme solutions are fairly close to each other (less than $1.1 \%$ over the whole transient), the difference with full-core diffusion is significant: the peak power in the latter case is around $11 \%$ higher and occurs $0.05 \mathrm{~s}$ earlier. This difference can be explained by two factors: (i) the cross-section in the experiment regions are different (since they are only SPH corrected for the full-core diffusion calculation) and (ii) the numerical scheme in the experiment region does have an impact on the control rod reactivity worth. Table 2 shows the worth of the control rod is indeed about $105 \mathrm{pcm}$ higher in the full-core diffusion calculation. The difference in the curves is consistent with that difference in reactivity. In particular, it makes sense that the diffusion power profile reaches a higher peak power (since the reactivity insertion is larger) and that the peak occurs before (since the temperature also increases faster and thus the feedback becomes more important earlier). 


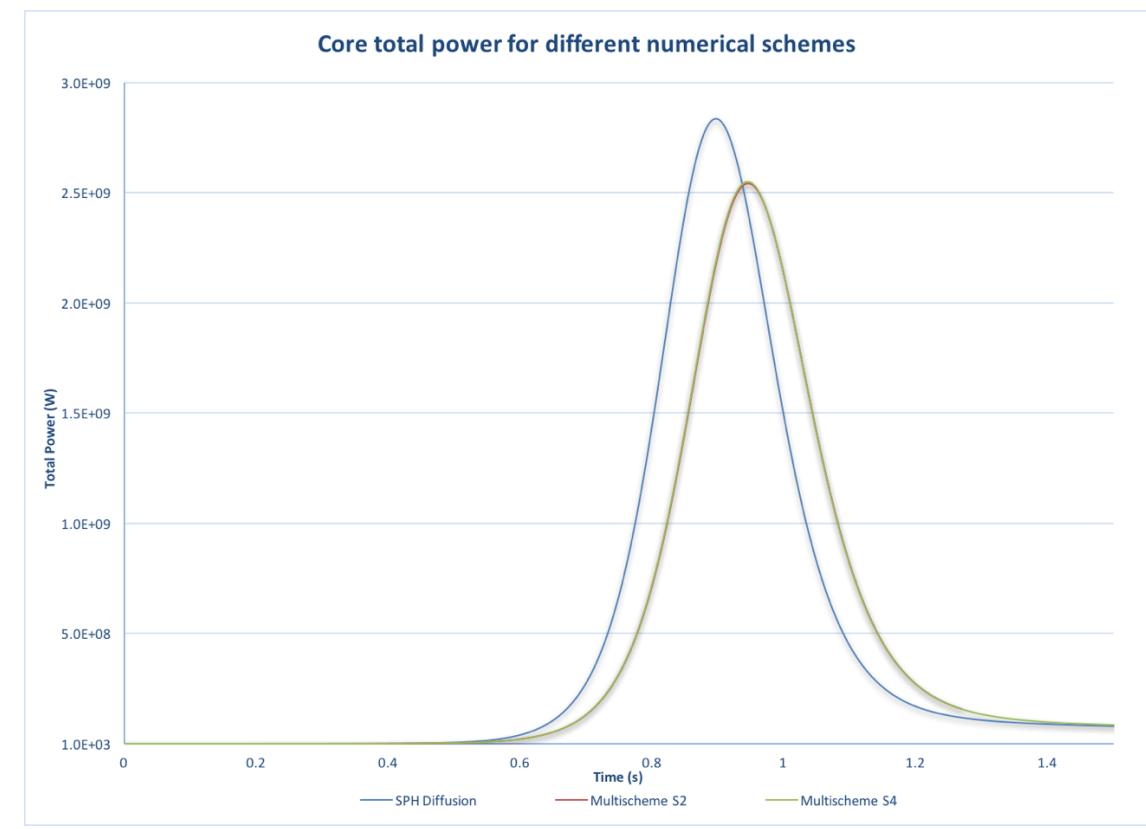

Figure 7: Power profile in the TREAT core as a function of time.

Table 2: Control rod (CR) worth for full-core SPH diffusion and multischeme SPHdiffusion/SAAF-S2. The first and second columns indicate the eigenvalue for the CR inserted and withdrawn, respectively.

\begin{tabular}{|c|cc|c|}
\hline & Eigenvalue (CR in) & Eigenvalue (CR out) & CR worth $(\rho=\Delta k / k)$ \\
\hline SPH-Diffusion & 0.99135 & 1.01440 & $2325 \mathrm{pcm}$ \\
\hline Multischeme SAAF-SN/Diffusion & 1.01109 & 1.03353 & $2220 \mathrm{pcm}$ \\
\hline
\end{tabular}

\subsubsection{Discussion}

This result is significant because it tends to show that the Multi-SERTTA experiment does have a non-negligible impact on the rest of the core. In particular, it was shown that replacing the experiment with air changed the core reactivity by about $175 \mathrm{pcm}$ (with Serpent calculations), mostly because of the water and steel surrounding the fuel stacks in the experiment. While it was originally thought that calculations decoupling the experiment region and the rest of the core would allow to reduce the complexity of the problem, 
this would indicate that this approach is not feasible for experiment like Multi-SERTTA. Rather, the multischeme methodology should prove invaluable to tackle such problems and optimize computational effort. However, it is noted that equivalence techniques might be necessary to implement for multischeme to ensure that reactivity insertion during transient is extremely accurate, lest the peak power - and thus the energy deposited in each experiment - be mispredicted.

\section{Conclusions and Future Work}

We discuss the interface conditions with the upwinding method for multischeme transport calculations in Rattlesnake. All five type of interface conditions for coupling eight different discretization schemes available in Rattlesnake are detailed. Results of its application to TREAT experiment modeling are presented. The designs on the user interface and preconditioning for multischeme transport are also briefly presented. We conclude that multischeme transport capability in Rattlesnake is up running. However, we emphasize that the accuracy of modeling and simulation relies on how different schemes are chosen, how they are assigned to mesh blocks and how the cross section data are generated and homogenization equivalence are done. Rattlesnake provides a whole new dimension of capabilities with multischeme transport, and it will be up to users to create the model to best simulate their targeting problem. The current multischeme transport capability can be enhanced in several aspects. The group prolongation can be added for coupling schemes with different numbers of energy groups. Better preconditioning than the current split preconditioning could be desired. Combining user supplied preconditioners with the preconditioners within Rattlesnake can be done. Nonlinear diffusion acceleration or diffusion synthetic acceleration requires more work for multischeme transport. Homogenization equivalence could be combined with multischeme transport for generating better cross section data and improve the overall accuracy of modeling and simulations. We can also add LS schemes into multischeme transport calculations. These enhancements can be pursued in the future when the needs from analysis tasks come.

\section{ACKNOWLEDGMENTS}

This work is supported by the U.S. Department of Energy, under DOE Idaho Operations Office Contract DE-AC07-05ID14517. Accordingly, the U.S. Government retains a nonex- 
clusive, royalty-free license to publish or reproduce the published form of this contribution, or allow others to do so, for U.S. Government purposes. 


\section{References}

[1] Yaqi Wang et al. "Hybrid PN - SN with Lagrange multiplier and upwinding for the multiscale transport capability in Rattlesnake". In: Progress in Nuclear Energy (Apr. 2017). DOI: $10.1016 / j$.pnucene.2017.03.020.

[2] M. M. Nanneh and M. M. R. Williams. "A Diffusion-Transport Theory Hybrid Method for Calculating Neutron Flux Distributions in Slab Lattices". In: Atomkernenergie Kerntechnik 47 (1985), pp. 221-224.

[3] Ricardo C. Barros et al. "Progress in Spectral Nodal Methods Applied to Discrete Ordinates Transport Problems". In: Progress in Nuclear Energy 33.1/2 (1998), pp. 117 154.

[4] Enrico Girardi and Jean-Michel Ruggieri. "Mixed First- and Second-Order Transport Method Using Domain Decomposition Techniques for Reactor Core Calculations". In: Proc. International Conference on Supercomputing in Nuclear Application. Paris, France, 2003.

[5] Enrico Girardi et al. "A New Method for the Treatment of Local Strong Heterogeneities and its Application to the Phebus Experimental Facility". In: PHYSOR 2004 - The Physics of Fuel Cycles and Advanced Nuclear Systems : Global Developments. American Nuclear Society, Lagrange Park, IL. Chicago, Illinois, Apr. 2004.

[6] Dmitriy Y. Anistratov and Nicholas D. Stehle. "Computational Transport Methodology Based on Decomposition of a Problem Domain into Transport and Diffusive Subdomains". In: Journal of Computational Physics 231 (2012), pp. 8009-8028.

[7] Sergiy Manolov, Jim E. Morel, and Cristian Rabiti. "Hybrid Sn-Diffusion and Sn-P3 Transport Calculations". In: International Conference on Mathematics and Computational Methods Applied to Nuclear Science and Engineering. Sun Valley, Idaho, May 2013, pp. 2748-2758.

[8] Nicholas D. Stehle, Dmitriy Y. Anistratov, and Marvin L. Adams. "A hybrid transportdiffusion method for 2D transport problems with diffusive subdomains". In: Journal of Computational Physics 270 (Aug. 2014), pp. 325-344.

[9] K. S. Smith. "Assembly Homogenization Techniques for Light Water Reactor Analysis". In: Progress in Nuclear Energy 17,3 (July 1986), pp. 303-335.

[10] Yaqi Wang, Sebastian Schunert, and Vincent Laboure. Rattlesnake Theory Manual. INL. 2017. 
[11] Derek Gaston et al. "MOOSE: A Parallel Computational Framework for Coupled Systems of Nonlinear Equations". In: Nuclear Engineering and Design 239.10 (2009), pp. $1768-1778$.

[12] Derek R. Gaston et al. "Physics-based multiscale coupling for full core nuclear reactor simulation". In: Annals of Nuclear Energy 84 (2015), pp. 45-54. DOI: 10.1016/ j.anucene.2014.09.060.

[13] J.D. Hales et al. "Advanced multiphysics coupling for LWR fuel performance analysis". In: Annals of Nuclear Energy 84.1 (2015), pp. 98-110.

[14] F.N. Gleicher et al. "The Coupling of the Neutron Transport Application Rattlesnake to the Nuclear Fuels Application BISON under the MOOSE Framework". In: PHYSOR 2014 - The Role of Reactor Physics Towards a Sustainable Future. The Westin Miyako, Kyoto, Japan, Sept. 2014.

[15] F.N. Gleicher et al. "The Application of MAMMOTH for a Detailed Tightly Coupled Fuel Pin Simulation with a Station Blackout”. In: Top Fuel 2016 - LWR Fuels with Enhanced Safety Performance. Boise, ID, USA, Sept. 2016.

[16] Y. Wang et al. "Demonstration of MAMMOTH Fully-Coupled Multiphysics Simulation with the Godiva Benchmark Problem". In: M\&C 2017 - International Conference on Mathematics \& Computational Methods Applied to Nuclear Science \& Engineering. Jeju, Korea, 2017.

[17] Thomas G. Saller, Randal S. Baker, and Jon A. Dahl. "On-The-Fly Multigroup Weighting in PARTISN". In: $M \& C 2017$ - International Conference on Mathematics \& Computational Methods Applied to Nuclear Science \& Engineering. Jeju, Korea, Apr. 2017.

[18] Yaqi Wang, Hongbin Zhang, and Richard Martineau. "Diffusion Acceleration Schemes for the Self-Adjoint Angular Flux Formulation with a Void Treatment". In: Nuclear Science and Engineering 176 (2014), pp. 201-225.

[19] Yaqi Wang and Frederick N. Gleicher. "Revisit Boundary Conditions for the SelfAdjoint Angular Flux Formulation". In: PHYSOR 2014 - The Role of Reactor Physics toward a Sustainable Future. The Westin Miyako, Kyoto, Japan, Sept. 2014.

[20] H Hammer, J.E. Morel, and Y. Wang. "Nonlinear Diffusion Acceleration in Voids for the Weighted Least-Square Transport Equation". In: $M \& C$ 2017, International Conference on Mathematics \& Computational Methods Applied to Nuclear Science \& Engineering. ANS. Jeju, Korea, 2017. 
[21] Vincent M. Laboure, Yaqi Wang, and Mark D. DeHart. "Least-Squares $\mathrm{P}_{N}$ Formulation of the Transport Equation Using Self-Adjoint-Angular-Flux Consistent Boundary Conditions." In: PHYSOR 2016 - Unifying Theory and Experiments in the $21^{\text {st }}$ Century. ANS. Sun Valley, ID, May 2016.

[22] Sebastian Schunert et al. "A flexible nonlinear diffusion acceleration method for the $\{\mathrm{SN}\}$ transport equations discretized with discontinuous finite elements". In: Journal of Computational Physics 338 (June 2017), pp. 107-136. ISSN: 0021-9991. DOI: $10.1016 / j \cdot j c p .2017 .01 .070$. URL: http://wWW. sciencedirect.com/ science/article/pii/S0021999117301286.

[23] Vincent M. Laboure and Ryan G. McClarren. "Energy-Dependent Implicit Filtered $\mathrm{P}_{N}$ Method for Thermal Radiation Transport". In: International Conference on Mathematics and Computational Methods Applied to Nuclear Science and Engineering (M\&C 2017). American Nuclear Society (ANS). Jeju, Korea, Apr. 2017.

[24] Weixiong Zheng, Ryan G. McClarren, and Jim E. Morel. "An Accurate Globally Conservative Subdomain Discontinuous Least-Squares Scheme for Solving Neutron Transport Problems". In: Nuclear Science and Engineering (Dec. 2017), pp. 1-13. DOI: $10.1080 / 00295639.2017 .1407592$

[25] Vincent M. Laboure, Ryan G. McClarren, and Cory D. Hauck. "Implicit Filtered $\mathrm{P}_{N}$ for High-Energy Density Thermal Radiation Transport using Discontinuous Galerkin Finite Elements." In: Journal of Computational Physics 321 (2016), pp. 624-643. DOI: $10.1016 / j \cdot j c p .2016 .05 .046$, arXiv: 1601.08242 [physics.comp-ph], URL: http://www.sciencedirect.com/science/article/pii/S0021999116301917.

[26] Vincent M. Laboure. "Improved Fully-Implicit Spherical Harmonics Methods for First and Second Order Forms of the Transport Equation using Galerkin Finite Element". PhD thesis. Texas A\&M University, 2016.

[27] D. N. Arnold. "An interior penalty finite element method with discontinuous elements". In: SIAM Journal on Numerical Analysis 19 (1982), pp. 742-760.

[28] S. Chandrasekhar. Radiative Transfer. Dover Publications Inc., Mar. 28, 2003. 416 pp. ISBN: 0486605906. URL: http: / / www . ebook . de / de / product / 3303044/s_ chandrasekhar_radiative_transfer.html.

[29] S. Balay et al. PETSc Users Manual. 3.6. Mathematics and Computer Science Division, Argonne National Laboratory. June 2015.

[30] J. Leppänen. Serpentfffdfffdffd a Continuous-energy Monte Carlo Reactor Physics Burnup Calculation Code. Tech. rep. VTT Technical Research Centre of Finland, 2015. 